Behavioral/Systems/Cognitive

\title{
Human Striatal Response to Salient Nonrewarding Stimuli
}

\author{
Caroline F. Zink, ${ }^{1}$ Giuseppe Pagnoni, ${ }^{1}$ Megan E. Martin, ${ }^{1}$ Mukeshwar Dhamala, ${ }^{1,2}$ and Gregory S. Berns ${ }^{1,3}$ \\ ${ }^{1}$ Department of Psychiatry and Behavioral Sciences, Emory University School of Medicine, Atlanta, Georgia 30322, and ${ }^{2}$ School of Physics and ${ }^{3}$ Department \\ of Biomedical Engineering, Georgia Institute of Technology, Atlanta, Georgia 30332
}

\begin{abstract}
Although one proposed function of both the striatum and its major dopamine inputs is related to coding rewards and reward-related stimuli, an alternative view suggests a more general role of the striatum in processing salient events, regardless of their reward value. Here we define saliency as an event that both is unexpected and elicits an attentional-behavioral switch (i.e., arousing). In the present study, human striatal responses to nonrewarding salient stimuli were investigated. Using functional magnetic resonance imaging (fMRI), the blood oxygenation level-dependent signal was measured in response to flickering visual distractors presented in the background of an ongoing task. Distractor salience was manipulated by altering the frequency of distractor occurrence. Infrequently presented distractors were considered more salient than frequently presented distractors. We also investigated whether behavioral relevance of the distractors was a necessary component of saliency for eliciting striatal responses. In the first experiment (19 subjects), the distractors were made behaviorally relevant by defining a subset of them as targets requiring a button press. In the second experiment (17 subjects), the distractors were not behaviorally relevant (i.e., they did not require any response). The fMRI results revealed increased activation in the nucleus accumbens after infrequent (high salience) relative to frequent (low salience) presentation of distractors in both experiments. Caudate activity increased only when the distractors were behaviorally relevant. These results demonstrate a role of the striatum in coding nonrewarding salient events. In addition, a functional subdivision of the striatum according to the behavioral relevance of the stimuli is suggested.
\end{abstract}

Key words: fMRI; striatum; predictability; salience; reward; behavioral relevance

\section{Introduction}

The striatum has been implicated in various functions, both motor and cognitive, but the exact nature of its functions remains essentially unknown. Decades of animal research suggest that both the striatum and its phasic dopaminergic input play an important role in coding rewards and reward-associated stimuli (for review, see Schultz, 1998; Schultz et al., 2000). Recent neuroimaging studies in humans support these claims (Delgado et al., 2000; Knutson et al., 2000, 2001a,b; Berns et al., 2001, Breiter et al., 2001; Pagnoni et al., 2002; Elliott et al., 2003). An alternative view contends that rather than specifically processing rewardrelated stimuli, activity within the striatum (including its major dopaminergic inputs) codes all salient events, including and extending beyond rewards. Salience here refers to any unexpected stimuli or environmental changes that are either arousing or that elicit an attentional-behavioral switch (Redgrave et al., 1999; Horvitz, 2000). Evidence for this view comes from studies showing that dorsal (Rolls et al., 1983; Caan et al., 1984; Hikosaka et al., 1989; Ravel et al., 1999; Shimo and Hikosaka, 2001) and ventral (Williams et al., 1993; Setlow et al., 2003) striatal neurons respond to such salient stimuli, including arousing, aversive, novel, and behaviorally relevant events, especially when unexpected. Furthermore, the presentation of salient nonrewarding stimuli in

Received May 5, 2003; revised July 10, 2003; accepted July 16, 2003.

This work was supported by National Institutes of Health Grants R01 EB002635 and K08 DA00367 to G.S.B.

Correspondence should be addressed to Dr. Gregory S. Berns, Department of Psychiatry and Behavioral Sciences, 1639 Pierce Drive, Woodruff Memorial Research Building Suite 4000, Atlanta, GA 30322. E-mail: gberns@emory.edu. Copyright $\odot 2003$ Society for Neuroscience $\quad$ 0270-6474/03/238092-06\$15.00/0 several modalities increases dopamine levels in the striatum and increases midbrain dopaminergic neuron firing (for review, see Horvitz, 2000). These findings link the striatum with processing salient events because nearly all striatal cells are innervated by midbrain dopaminergic projections (Groves et al., 1995). Outside of a rewarding context, striatal processing of salient events is mostly unexplored in humans. A few human neuroimaging studies have revealed increased striatal activity in response to both punishment (Knutson et al., 2000, 2003) and aversive stimuli (Becerra et al., 2001); however, not every investigation of punishment in humans has found increased striatal activity (Delgado et al., 2000). Such conflicting results suggest that either behavioral context or stimulus magnitude plays an important role in modulating striatal function. To our knowledge, the role of the striatum in processing neutral salient stimuli has not been investigated specifically in humans.

Using functional magnetic resonance imaging (fMRI), we sought to investigate how human striatal (caudate, putamen, and nucleus accumbens) activity was modulated by neutral salient events. In the present experiments, flickering visual distractors were presented outside subjects' focus of attention. Distractor salience was manipulated by altering the frequency of distractor occurrence. Less frequent events are more salient because their occurrence is inherently less predictable than that of frequent ones. We performed two experiments to determine whether the stimuli had to be behaviorally relevant rather than just innately arousing to elicit striatal activation. In the first experiment, the distractors were behaviorally relevant in that they potentially required a response, whereas in the second experiment, the distrac- 
A.
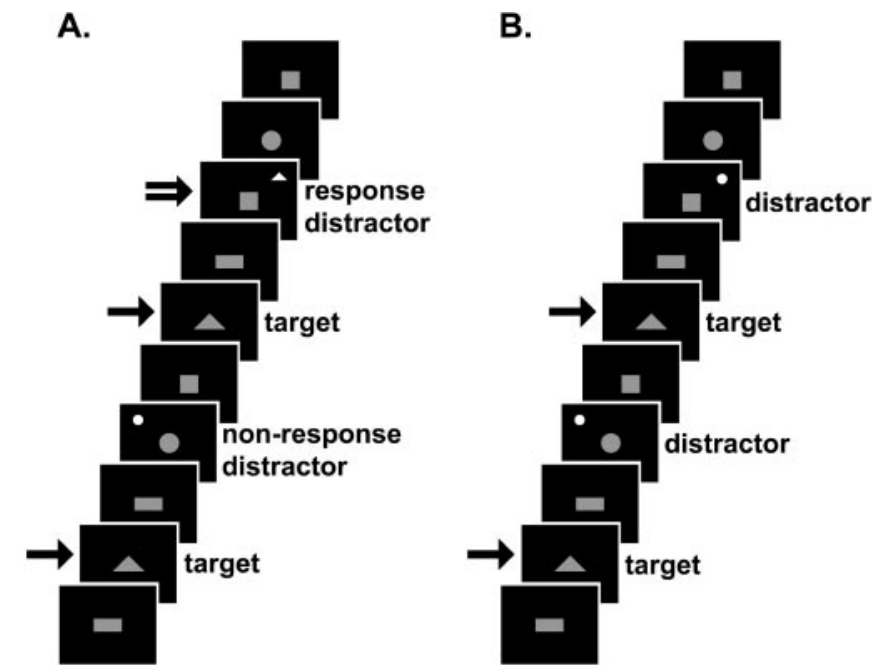

Figure 1. Schematic of tasks. Each stimulus appeared for $750 \mathrm{msec}$ (ISI, $2000 \mathrm{msec}$ ). The single-tailed arrows indicate a button 1 press; the double-tailed arrow indicates a button 2 press. $A$, Excerpt from experiment 1, behaviorally relevant distractors, frequent run. The white distractors appear as circles or triangles flickering in the background of the ongoing task. Subjects were instructed to press button 1 when a blue (shown here in gray) central triangle appeared and button 2 when the white distractor in the background was a triangle. $B$, Excerpt from experiment 2 , behaviorally irrelevant distractors, frequent run. The white distractors appeared as circles flickering in the background of the ongoing task. Subjects were instructed to press button 1 when a blue (shown here in gray) central triangle appeared. No responses were made to the distractors. In both experiments, the distractors appeared randomly in time and space. Any patterns detected in the figure are purely coincidental. The infrequent runs of both experiments (data not shown) were the same, except that the distractors occurred less often.

tors were behaviorally irrelevant by never requiring a response. We hypothesized that a greater fMRI signal would be measured in the striatum to the infrequent (high salience) relative to frequent (low salience) distractors and that the striatal activity would be modulated by behavioral relevance.

\section{Materials and Methods}

Subjects. Thirty-six right-handed, healthy adults (19 females, 17 males), ages 19-44, were included in the study, 19 in experiment 1 and 17 in experiment 2. Given the similarity of the two experiments, two separate samples of subjects were recruited for the experiments to avoid testretest confounds. Subjects in both groups were sampled from the same population (recruited from the Emory University community, demographically similar between groups, with no significant differences in ages between groups). Subjects had no history of neurological or psychiatric disorders and gave informed consent for a protocol approved by the Emory University Institutional Review Board.

Experimental tasks. All stimuli presentations and recordings of reaction times were performed with Presentation software (Neurobehavioral Systems, Inc., San Francisco, CA).

Experiment 1 is diagramed in Figure $1 \mathrm{~A}$. While in the scanner, subjects viewed one of four blue shapes (square, rectangle, circle, or triangle) presented in random order in the center of a black screen for $750 \mathrm{msec}$ within a $2000 \mathrm{msec}$ interstimulus interval (ISI). Subjects were told that they would see blue shapes, one at a time, in the center of a screen. They were instructed to press button 1 using their right index finger each time a triangle (target stimuli) appeared. The other shapes were designated as nontargets. In a subset of the nontargets, a salient distractor in the form of a smaller, white, flickering $(28 \mathrm{~Hz})$ circle or triangle appeared in the background (in one of four screen corner locations randomly). Subjects were told that a flickering white shape would occasionally appear in the background of the ongoing task and were instructed to press button 2 using their right middle finger only when the distractor was a triangle (response distractor). They were not required to react to the circle distractors (nonresponse distractors). Both distractor types (response and nonresponse) were behaviorally relevant in that they potentially required a response. As such, the distractors elicited a momentary attentional and behavioral switch. A single experimental session consisted of four runs lasting $240 \mathrm{sec}$ each, with 116 stimuli presentations. In each run, the central triangle target stimulus appeared 29 times.

The salience of the distractor stimuli was manipulated by changing their frequency of occurrence; the greater the frequency, the more likely that a given trial would contain a distractor, thus greater frequency was associated with greater predictability on average and less salience. In one run, a distractor stimulus appeared 25 times (frequent), with 1-4 (average, 2.9) stimuli between consecutive distractor stimuli. During the other three runs, a distractor stimulus only occurred 4 times (infrequent), with 24-32 (average, 28.5) stimuli between consecutive distractor stimuli. By having three infrequent runs, there were enough infrequent distractor stimuli to perform a statistical analysis of adequate power. In all runs, the distractor stimuli occurred irregularly in time and never at the same time as a central triangle target. Subjects were not instructed to fixate and were free to move their eyes. Run order was counterbalanced across subjects.

Experiment 2 is diagramed in Figure $1 B$. A separate group of subjects performed the same task as described above, except for the following differences: the distractors were all circles and behaviorally irrelevant in that they never required a response. Subjects were told that they would see blue shapes, one at a time, in the center of a screen and were instructed to press button 1 using their right index finger each time a triangle appeared. In experiment 2 , the distractors were not mentioned to the subjects when instructions were given. Again, the salience of the distractors was manipulated by changing their frequency of occurrence. Although not behaviorally relevant, the infrequent distractors were considered salient by virtue of their innate arousing properties (i.e., stark color contrast and flickering).

fMRI imaging. Scanning was performed on a 1.5 Tesla Philips Intera scanner (Eindhoven, The Netherlands). For each subject, a T1-weighted structural image was acquired for anatomical reference, followed by four whole-brain functional runs of 120 scans each to measure the $\mathrm{T}^{*}$ weighted blood oxygenation level-dependent (BOLD) effect (gradientrecall echo-planar imaging; repetition time, $2000 \mathrm{msec}$; echo time, 40 msec; flip angle, $90^{\circ}$; $64 \times 64$ matrix; field of view, $240 \mathrm{~mm} ; 245 \mathrm{~mm}$ axial slices acquired parallel to the anteroposterior commissural line). Head movement was minimized with padding.

fMRI analysis. The data were analyzed using statistical parametric mapping (SPM99) (Friston et al., 1995b). For each subject, the first 12 scans in each run were excluded from the analysis to discount artifacts related to the transient phase of magnetization. Slice timing correction was used to adjust for time differences resulting from multislice imaging acquisition. Motion correction to the first functional scan was performed within subjects using a six-parameter rigid-body transformation. Each individual's anatomical image was co-registered to the mean of their functional images using a rigid-body transformation and then spatially normalized to the Montreal Neurological Institute (MNI) template conforming to the Talairach orientation system (Talairach and Tournoux, 1988) by applying a 12-parameter affine transformation followed by nonlinear warping using basis functions (Ashburner and Friston, 1999). The computed transformation parameters were applied to all of the functional images, interpolating to a final voxel size of $4 \times 4 \times 4 \mathrm{~mm}^{3}$. Images were subsequently spatially smoothed with an $8 \mathrm{~mm}$ isotropic Gaussian kernel and temporally filtered with a synthetic hemodynamic responsesmoothing function to attenuate high-frequency components of the signal resulting from noise.

A random-effects, event-related statistical analysis was performed with SPM99 (Friston et al., 1995a, 1999) in a two-level procedure for each experiment. For experiment 1 , at the first level, a separate general linear model was specified for each subject. The BOLD responses to four event types (nontargets, targets, response distractors, and nonresponse distractors) for each run were modeled with a basis function consisting of a synthetic hemodynamic response function (consisting of two gamma functions shifted $2 \mathrm{sec}$ apart) and its first order temporal derivative. A contrast image was calculated for each subject corresponding to the main effect of distractor frequency: infrequent distractors greater than frequent distractors (regardless of type). The individual contrast images 
were then entered into a second-level analysis using a one-sample $t$ test. The resulting summary statistical map was thresholded at $p<$ 0.05 (uncorrected for multiple comparisons), and then a small volume correction (SVC) was applied to six $6 \mathrm{~mm}$ radius spherical regions of interest (ROIs). Because of the a priori hypothesis concerning the striatum, the ROIs were centered at the following locations (MNI coordinates): right nucleus accumbens, $12,8,-8$; left nucleus accumbens, $-12,8,-8$; right caudate, 12, 8, 12; left caudate, $-12,8,12$; right putamen, 24, 4, 4; and left putamen, $-24,4,4$ (Fig. 2). The coordinates corresponding to each ROI were based on those specified by the Talairach Daemon database (Lancaster et al., 2000), as implemented in AFNI software (Cox, 1996), which was also used to convert the Talairach coordinates to the corresponding MNI coordinates. Small volume correction is a re-

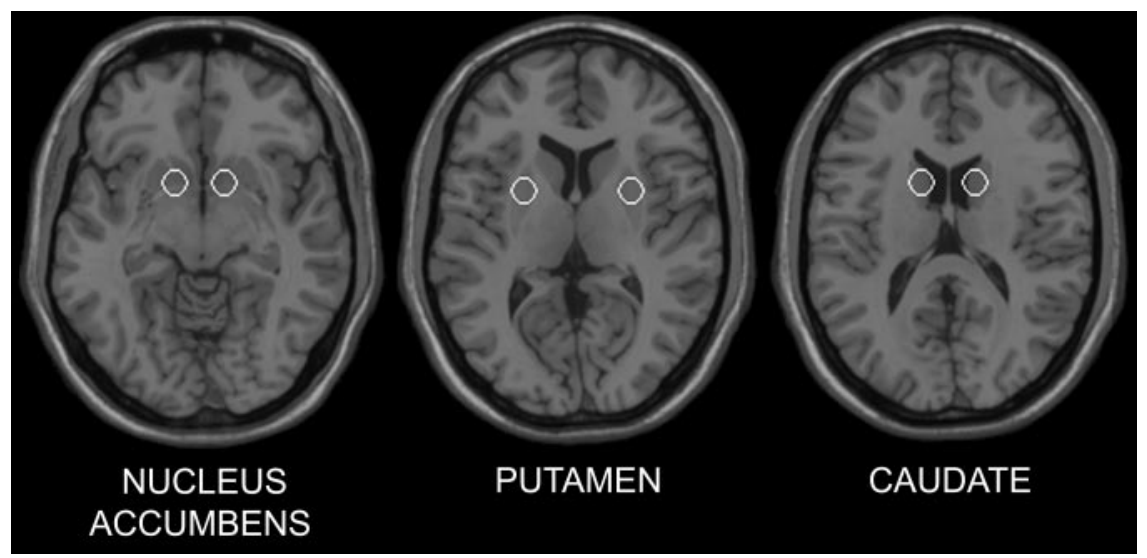

Figure 2. The locations of the following bilateral ROls: nucleus accumbens, putamen, and caudate. The ROls are shown on axial sections of a structural template MRI. stricted application of the random Gaussian field theory to account for multiple comparisons when statistical inference is limited to an a priori specified region of interest (Worsley et al., 1996). For each subject, the parameter estimates (i.e., effect size expressed as percentage of the global mean intensity of the scans) of each distractor type (response and nonresponse) in the two conditions (frequent and infrequent) were averaged across voxels within the specified ROIs that survived $p_{\text {SVC }}<0.05$ for the contrast, infrequent distractors greater than frequent distractors. Because the infrequent distractors resulted in greater activation than the frequent distractors, we performed a post hoc assessment of the individual contributions of the response and nonresponse distractors to the infrequent condition. This was tested with a pairwise comparison on the parameter estimates for the nonresponse versus response distractors in the infrequent condition. The same analysis for experiment 1 was conducted for experiment 2, except that because there was only one distractor type (nonresponse) in experiment 2 , three rather than four event types were modeled: nontargets, targets, and distractors. The assessment of the individual contributions of response and nonresponse distractors that was performed in experiment 1 did not apply in this second experiment.

In a separate analysis, the first-level individual contrast images from experiments 1 and 2 were entered into a second-level analysis using a two-sample $t$ test to assess the interaction of behavioral relevance with saliency. Because the subjects in each experiment were sampled from the same population (i.e., two different groups of individuals from the same population), any differences between these groups could be attributed to differences in tasks (i.e., distractor behavioral relevance). For each interaction contrast, (1) (infrequent distractors - frequent distractors) $\times$ (relevant - irrelevant) and (2) (infrequent distractors - frequent distractors $) \times$ (irrelevant - relevant), the summary statistical map was thresholded at $p<0.05$ and then an SVC was applied to the same six 6 $\mathrm{mm}$ radius spherical ROIs specified in the one-sample analyses above.

\section{Results}

In both experiments, subjects made an average of less than one error per run. In experiment 1 , the reaction times to the response distractors were significantly longer for the infrequently presented distractors (mean $\pm \mathrm{SE}=683 \pm 27 \mathrm{msec}$ ) than for the frequently presented distractors (mean $\pm \mathrm{SE}=623 \pm 24 \mathrm{msec})(p<0.001$; paired $t$ test $)$. Reaction times to the central triangle targets in the frequent run (mean $\pm \mathrm{SE}=598 \pm 20 \mathrm{msec}$ ) and infrequent runs (mean $\pm \mathrm{SE}=$ $579 \pm 19 \mathrm{msec}$ ) were also significantly different in experiment 1 ( $p=0.008$; paired $t$ test). In experiment 2 , there was no significant difference in reaction times to the central triangle targets between the frequent run (mean $\pm \mathrm{SE}=468 \pm 17 \mathrm{msec})$ and infrequent runs (mean $\pm \mathrm{SE}=456 \pm 14 \mathrm{msec})(p=0.132$; paired $t$ test $)$.

The fMRI ROI results for the contrast, infrequent distractors greater than frequent distractors, in experiment 1 and

experiment 2 are summarized in Table 1. In experiment 1 (behaviorally relevant distractors), of the six striatal ROIs investigated, significant activations occurred bilaterally in the caudate and nucleus accumbens. Subjects' parameter estimates for each distractor type in the bilateral caudate and nucleus accumbens ROIs (putamen ROIs did not contain significantly activated voxels) revealed that in all four clusters, the greatest signal was measured for the infrequent nonresponse distractors compared with the other distractor types, including the infrequent response distractors (Fig. 3). For experiment 2, only the left nucleus accumbens ROI contained significant activation.

The results of the fMRI analysis comparing behavioral relevance between the two experiments are summarized in Table 2. For the interaction (infrequent distractors - frequent distractors $) \times($ relevant - irrelevant), significant activation occurred in the caudate bilaterally. No other ROI had activations. The interaction (infrequent distractors - frequent distractors) $\times$ (irrelevant - relevant) did not result in any significantly activated ROIs.

\section{Discussion}

Using fMRI, the present study investigated how human striatal activity was modulated by the saliency of nonrewarding stimuli. Stimuli considered salient must be unexpected and arousing (i.e., draw attention). In the present study, the subjects performed an ongoing continuous performance task designed to focus their attention, but brain activity linked to performing this central task was not of interest. The stimuli of interest were distractor stimuli flickering in the background of some of the nontargets in the central task. Saliency was manipulated across two dimensions: frequency (more frequent, less salient) and behavioral relevance.

An important feature of our tasks was the manipulation of distractor stimuli expectation through frequency. In each experiment, the frequency of distractor occurrence was modulated between runs to alter distractor expectation and thus saliency. When events occur more frequently, they become more expected and less salient. Infrequent stimuli with long intervals between consecutive events are unexpected and therefore more salient. Conforming to accepted definitions of infrequency (McCarthy et al., 1997; Clark et al., 2000; Kirino et al., 2000), infrequent distractors occurred in $<10 \%$ of the trials. Within each experiment, distractor properties, including location, exact timing, and type of distractor, were pseudorandomized in each run so that the sole manipulation of saliency came from relative frequency. In experiment 1 , the reaction times for infrequent distractors were signif- 
Table 1. Significantly activated $\left(p_{\mathrm{Svc}} \leq \mathbf{0 . 0 5}\right)$ striatal ROIs in experiments 1 and 2 for the contrast, infrequent distractors greater than frequent distractors

\begin{tabular}{|c|c|c|c|c|}
\hline Brain regions & $\begin{array}{l}\text { ROI center in MNI coordinates } \\
(x, y, z)\end{array}$ & $\begin{array}{l}\text { Cluster size } \\
\text { (voxels) }\end{array}$ & $\begin{array}{l}\text { Peak MNI coordinates } \\
(x, y, z)\end{array}$ & Peak Z score \\
\hline \multicolumn{5}{|c|}{ Experiment 1 (behaviorally relevant distractors) } \\
\hline Right caudate & $12,8,12$ & 14 & $12,12,16$ & 2.66 \\
\hline Left caudate & $-12,-8,12$ & 11 & $-12,12,16$ & 3.02 \\
\hline Right nucleus accumbens & $12,8,-8$ & 5 & $8,12,-8$ & 2.98 \\
\hline Left nucleus accumbens & $-12,8,-8$ & 18 & $-8,8,-12$ & 3.15 \\
\hline \multicolumn{5}{|c|}{ Experiment 2 (behaviorally irrelevant distractors) } \\
\hline Left nucleus accumbens & $-12,8,-8$ & 9 & $-8,12,-8$ & 3.01 \\
\hline
\end{tabular}

ROI, Region of interest; MNI, Montreal Neurological Institute; SVC, small volume correction.

A.

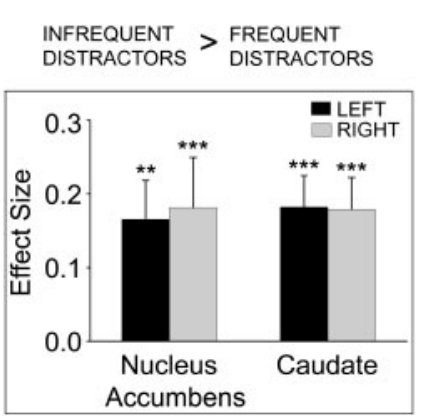

B.

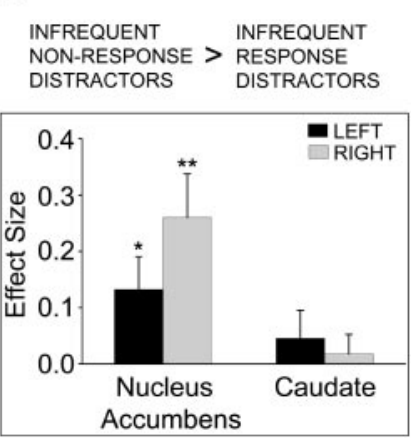

Figure 3. In experiment 1, single subjects' parameter estimates representing effect sizes (percentage of global mean intensity) were averaged across significantly activated voxels within the ROls for the contrast, infrequent distractors greater than frequent distractors. ROls with significant activations were bilateral nucleus accumbens and caudate. The contrast estimates are plotted for infrequent distractors relative to frequent distractors, regardless of response requirement $(A)$. Infrequent distractors were subdivided by response requirement, and contrast estimates for infrequent nonresponse distractors relative to infrequent response distractors are plotted $(B)$. Bar plots represent averages and SEs across subjects. ${ }^{*} p<0.05$; ${ }^{* *} p<$ $0.005{ }^{* * *} p<0.001$.

icantly longer than for frequent distractors, demonstrating a behavioral correlate of predictability.

In addition to predictability, the ability to preferentially draw attention contributes greatly to stimulus salience. This aspect of saliency either can be dependent on the behavioral context (i.e., on the basis of stimulus behavioral relevancy) or independent of behavioral context (i.e., on the basis of intrinsic stimulus traits) (Downar et al., 2002). In our experiments, we manipulated the behavioral context. In experiment 1 , the distractors were behaviorally relevant by virtue of a potential response. In experiment 2 , the distractors were behaviorally irrelevant because the distractors were unimportant for the task, and subjects did not have to respond to them. Subjects did report, however, that they "noticed" the distractors. When the distractors in experiment 2 were presented infrequently, they were still considered salient because of features independent of behavioral context, including their stark color contrast on the black background (as opposed to the less contrasting blue shapes in the ongoing task) and their flickering nature. In addition to the intrinsic properties of the stimuli, distractors in experiment 1 had an added dimension to their saliency as a result of their potential behavioral consequences. Importantly, both of the distractor types (response and nonresponse) in experiment 1 required subjects to momentarily interrupt the ongoing task, divert resources to the distractor, and possibly respond to it, although the button press only occurred to the response (triangle) distractors.

The results of the present study reveal that activity in the nucleus accumbens increased in response to unexpected, arousing changes in the visual environment (i.e., appearance of flickering distractors), whereas the caudate was recruited only when such a stimulus was behaviorally relevant. These patterns of activity are consistent with previous animal studies. Caudate neurons respond to cues and visual stimuli, but responses are not typically seen when these stimuli are independent of the task (Rolls et al., 1983). Aosaki et al. (1994) reported that neurons in the caudate do not respond to a cue before training; however, they do respond to the cue after learning that the cue signals upcoming reward and thus initiates licking. On the other hand, the nucleus accumbens responds to arousing visual stimuli, even when lacking task relevance (Williams et al., 1993). We observed no significant modulations of activity in the putamen, which is consistent with data suggesting that the putamen is most directly linked with motor control (Alexander and Crutcher, 1990) rather than salience per se.

From our data, we conclude that the human striatum (caudate and nucleus accumbens) plays a role in processing salient events, other than rewards, but the response is not homogeneous throughout the striatum. Redgrave et al. (1999) proposed that activity in the striatum provides a signal to switch attentional or behavioral resources, or both, to unexpected stimuli eliciting such responses. In accordance with this theory, our results suggest that the nucleus accumbens responds when an attentional switch is elicited, whereas the caudate responds when a behavioral switch is elicited.

An alternative interpretation may be that subjects found that responding to the distractors in experiment 1 was more rewarding than not responding to distractors in experiment 2. In this view, the differential striatal activation (caudate) would be caused by a difference in internal reward state. However, this interpretation is unlikely given that the infrequent nonresponse distractors, rather than the response distractors, elicited the greatest dorsal and ventral striatal activity. If the internal reward hypothesis were true, then not responding would have to be more rewarding than responding, a scenario that seems implausible. The greatest signal after infrequent nonresponse distractors also provides evidence that the striatal activity in experiment 1 was not attributable to motor execution (although its involvement in motor inhibition cannot be ruled out).

The interpretation of the present results relies on salience being manipulating through the alteration of distractor frequency. However, it should be noted in experiment 1 that the effect of frequency could be interpreted differently. Subjects had to switch between two stimulus-response channels both frequently and infrequently. The effect of frequency may therefore reflect differences in the rate of changing the response selection rather than salience. To our knowledge, the striatum has not been implicated in the coding rate of changing response selections, although future studies manipulating stimulus saliency in ways other than altering its frequency of occurrence could address this issue. 
Table 2. Striatal regions of interest differentially activated $\left(p_{\mathrm{svc}}<0.05\right)$ by behavioral relevance between the two experiments for the contrast, infrequent distractor greater than frequent distractors

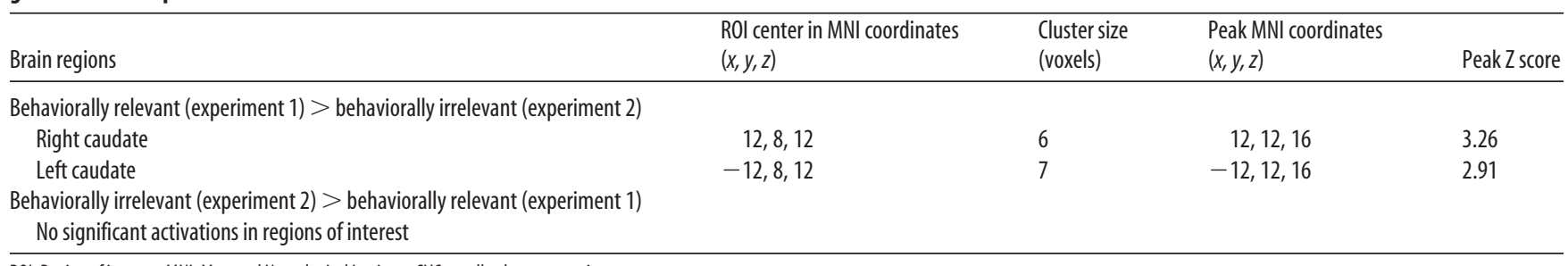

ROI, Region of interest; MNI, Montreal Neurological Institute; SVC, small volume correction.

Brain responses to infrequent salient stimuli have been assessed in other ways, such as oddball paradigms, but striatal activity has not been reported in these studies (McCarthy et al., 1997; Clark et al., 2000; Kirino et al., 2000; Casey et al., 2001). If the striatum codes unexpected events that elicit a switch of attention or behavior, as our results suggest, then oddball paradigms might not be effective for evoking striatal activations. In the aforementioned oddball paradigms, each stimulus appeared in the focus of attention so that no switch was elicited. By presenting the stimuli in the focus of attention, the subjects expected a stimulus to appear in a predictable location and typically at a predictable time. Although they were infrequent compared with other stimuli in the task, the stimuli of interest were not unexpected, as they would be if presented outside of the task. In the present study, we avoided these confounds by presenting the distractors outside of the focus of attention, thereby allowing us to modulate both stimulus expectation and behavioral relevance.

It should be noted that the signal measured in fMRI is an indirect measure of changes in cerebral blood flow, which tends to be more correlated with presynaptic activity than postsynaptic spiking (Logothetis et al., 2001). The BOLD signal cannot be associated directly with activity in specific cell types and is not a measurement of specific neurotransmitter release. We are unable to link the present results to specific neurons in the striatum [e.g., tonically active interneurons (TANs) or medium spiny projection neurons] or direct changes in dopamine transmission. However, because the BOLD signal is more correlated with presynaptic activity, the observed activations within the striatum probably do not represent spike rates of striatal projection neurons. TANs comprise $\sim 2 \%$ of all striatal cells (Pisani et al., 2001); therefore it is unlikely that the TANs are solely responsible for the reported changes in striatal activity. Dopaminergic inputs, which do respond to salient events (Horvitz, 2000), may interact with convergent glutamatergic cortical inputs in the striatum by amplifying strong (salient related) cortical inputs and dampening weak (nonsalient related) cortical inputs (Nicola et al., 2000; Horvitz, 2002). This interaction could be responsible for the signal changes observed in the striatum in our experiments. In the present study, although dopamine inputs innervate the entire striatum, differential striatal activation between experiments could have occurred as a result of the recruitment of different cortical inputs coding for behavioral relevance. The current analysis was restricted to the striatum; however, it would be interesting in future studies to examine which cortical areas are also recruited under similar task conditions.

In conclusion, the results of the present study extend the role of the striatum from reward processing to saliency processing. Specifically, our data suggest that the nucleus accumbens plays a role in coding unexpected arousing events, whereas caudate activity is more closely linked to the behavioral relevance of stimuli. This notion is not incompatible with the reward theory of the striatum. Both unexpected rewards and reward-related stimuli are salient by being behaviorally relevant, especially to a fooddeprived animal (as often is the case in the studies investigating reward processing), and ongoing behaviors must be interrupted to approach and consume rewards. The present study provides evidence that activity in the human striatum codes more than rewards or even stimuli potentially leading to rewards. Rather, the striatal system may have evolved more generally to subserve the processing of any salient stimulus.

\section{References}

Alexander GE, Crutcher MD (1990) Functional architecture of basal ganglia circuits: neural substrates of parallel processing. Trends Neurosci 13:266-271.

Aosaki T, Tsubokawa H, Ishida A, Watanabe K, Graybiel AM, Kimura M (1994) Responses of tonically active neurons in the primate's striatum undergo systematic changes during behavioral sensorimotor conditioning. J Neurosci 14:3969-3984.

Ashburner J, Friston KJ (1999) Nonlinear spatial normalization using basis functions. Hum Brain Mapp 7:254-266.

Becerra L, Breiter HC, Wise R, Gonzalez RG, Borsook D (2001) Reward circuitry activation by noxious thermal stimuli. Neuron 32:927-946.

Berns GS, McClure SM, Pagnoni G, Montague PR (2001) Predictability modulates human brain response to reward. J Neurosci 21:2793-2798.

Breiter HC, Aharon I, Kahneman D, Dale A, Shizgal P (2001) Functional imaging of neural responses to expectancy and experience of monetary gains and losses. Neuron 30:619-639.

Caan W, Perrett DI, Rolls ET (1984) Responses of striatal neurons in the behaving monkey. 2. Visual processing in the caudal neostriatum. Brain Res 290:53-65.

Casey BJ, Forman SD, Franzen P, Berkowitz A, Braver TS, Nystrom LE, Thomas KM, Noll DC (2001) Sensitivity of prefrontal cortex to changes in target probability: a functional MRI study. Hum Brain Mapp 13:26-33.

Clark VP, Fannon S, Lai S, Benson R, Bauer L (2000) Responses to rare visual target and distractor stimuli using event-related fMRI. J Neurophysiol 83:3133-3139.

Cox RW (1996) AFNI: software for analysis and visualization of functional magnetic resonance neuroimages. Comput Biomed Res 29:162-173.

Delgado MR, Nystrom LE, Fissell C, Noll DC, Fiez JA (2000) Tracking the hemodynamic responses to reward and punishment in the striatum. J Neurophysiol 84:3072-3077.

Downar J, Crawley AP, Mikulis DJ, Davis KD (2002) A cortical network sensitive to stimulus salience in a neutral behavioral context across multiple sensory modalities. J Neurophysiol 87:615-620.

Elliott R, Newman JL, Longe OA, Deakin JFW (2003) Differential response patterns in the striatum and orbitofrontal cortex to financial reward in humans: a parametric functional magnetic resonance imaging study. J Neurosci 23:303-307.

Friston KJ, Frith CD, Turner R, Frackowiak RSJ (1995a) Characterizing dynamic brain responses with fMRI: a multivariate approach. NeuroImage 2:166-172.

Friston KJ, Holmes AP, Worsley KJ, Poline JB, Frith CD, Frackowiak RSJ (1995b) Statistical parametric maps in functional imaging: a general linear approach. Hum Brain Mapp 2:189-210.

Friston KJ, Holmes AP, Worsley KJ (1999) How many subjects constitute a study? NeuroImage 10:1-5.

Groves PM, Garcia-Munoz M, Linder JC, Manley MS, Martone ME, Young SJ 
(1995) Elements of the intrinsic organization and information processing in the neostriatum. In: Models of information processing in the basal ganglia (Houk JC, Davis LJ, Beiser DG, eds), pp 51-96. Cambridge, MA: MIT.

Hikosaka O, Sakamoto M, Usui S (1989) Functional properties of monkey caudate neurons II. visual and auditory responses. J Neurophysiol 61:799-813.

Horvitz JC (2000) Mesolimbocortical and nigrostriatal dopamine responses to salient non-reward events. Neuroscience 96:651-656.

Horvitz JC (2002) Dopamine gating of glutamatergic sensorimotor and incentive motivational input signals to the striatum. Behav Brain Res 137:65-74.

Kirino E, Belger A, Goldman-Rakic P, McCarthy G (2000) Prefrontal activation evoked by infrequent target and novel stimuli in a visual target detection task: an event-related functional magnetic resonance imaging study. J Neurosci 20:6612-6618.

Knutson B, Westdorp A, Kaiser E, Hommer D (2000) FMRI visualization of brain activity during a monetary incentive delay task. NeuroImage $12: 20-27$.

Knutson B, Adams CM, Fong GW, Hommer D (2001a) Anticipation of increasing monetary reward selectively recruits nucleus accumbens. J Neurosci 21:RC159(1-5).

Knutson B, Fong GW, Adams CM, Varner JL, Hommer D (2001b) Dissociation of reward anticipation and outcome with event-related fMRI. NeuroReport 12:3683-3687.

Knutson B, Fong GW, Bennett SM, Adams CM, Hommer D (2003) A region of mesial prefrontal cortex tracks monetarily rewarding outcomes: characterization with rapid event-related fMRI. NeuroImage 18:263-272.

Lancaster JL, Woldorff MG, Parsons LM, Liotti M, Freitas CS, Rainey L, Kochunov PV, Nickerson D, Mikiten SA, Fox PT (2000) Automated Talairach atlas labels for functional brain mapping. Hum Brain Mapp 10:120-131.

Logothetis NK, Pauls J, Augath M, Trinath T, Oeltermann A (2001) Neurophysiological investigation of the basis of the fMRI signal. Nature 412:150-157.

McCarthy G, Luby M, Gore J, Goldman-Rakic P (1997) Infrequent events transiently activate human prefrontal and parietal cortex as measured by functional MRI. J Neurophysiol 77:1630-1634.

Nicola SM, Surmeier DJ, Malenka RC (2000) Dopaminergic modulation of neuronal excitability in the striatum and nucleus accumbens. Annu Rev Neurosci 23:185-215.

Pagnoni G, Zink CF, Montague PR, Berns GS (2002) Activity in human ventral striatum locked to errors of reward prediction. Nat Neurosci 5:97-98.

Pisani A, Bonsi P, Picconi B, Tolu M, Giacomini P, Scarnati E (2001) Role of tonically-active neurons in the control of striatal function: cellular mechanisms and behavioral correlates. Prog Neuropsychopharmacol Biol Psychiatry 25:211-230.

Ravel S, Legallet E, Apicella P (1999) Tonically active neurons in the monkey striatum do not preferentially respond to appetitive stimuli. Exp Brain Res 128:531-534.

Redgrave P, Prescott TJ, Gurney K (1999) Is the short-latency dopamine response too short to signal reward error? Trends Neurosci 22:146-151.

Rolls ET, Thorpe SJ, Maddison SP (1983) Responses of striatal neurons in the behaving monkey. I. Head of the caudate nucleus. Behav Brain Res 7:179-210.

Schultz W (1998) Predictive reward signal of dopamine neurons. J Neurophysiol 80:1-27.

Schultz W, Tremblay L, Hollerman JR (2000) Reward processing in primate orbitofrontal cortex and basal ganglia. Cereb Cortex 10:272-284.

Setlow B, Schoenbaum G, Gallagher M (2003) Neural encoding in ventral striatum during olfactory discrimination learning. Neuron 38:625-636.

Shimo Y, Hikosaka O (2001) Role of tonically active neurons in primate caudate in reward-oriented saccadic eye movement. J Neurosci 21:7804-7814.

Talairach J, Tournoux P (1988) Co-planar stereotaxic atlas of the human brain. Stuttgart, Germany: Thieme.

Williams GV, Rolls ET, Leonard CM, Stern C (1993) Neuronal responses in the ventral striatum of the behaving macaque. Behav Brain Res 55:243-252.

Worsley KJ, Marrett S, Neelin P, Vandal AC, Friston KJ, Evans AC (1996) A unified statistical approach for determining significant signals in images of cerebral activation. Hum Brain Mapp 4:58-73. 